\title{
Ablation for atrial fibrillation: what are acceptable levels of experience, efficacy, and complications?
}

\author{
Kelley P. Anderson
}

Received: 31 January 2012 / Accepted: 6 February 2012 / Published online: 9 March 2012

(C) Springer Science+Business Media, LLC 2012

The 2011 ACCF/AHA/HRS Focused Update on the Management of Patients With Atrial Fibrillation upgraded the indication for atrial fibrillation (AF) catheter ablation from class IIa to class I (level of evidence A) for "selected patients with significantly symptomatic, paroxysmal AF who have failed treatment with an antiarrhythmic drug and have normal or mildly dilated left atria, normal or mildly reduced LV function, and no severe pulmonary disease" [1]. This implies that the members of the guideline committee agree that the benefit of AF catheter ablation outweighs the risks for this group of patients based on the highest level of evidence. The benefits are related to reduction of symptoms and improvement in quality of life. However, the procedure is not known to improve longevity or to reduce complications such as stroke or to eliminate the need for anticoagulation and attendant risks of bleeding complications. Moreover, the benefits are limited by the success rate of the procedure. In order to make an informed decision, patients, referring clinicians, and $\mathrm{AF}$ ablation operators need to have specific information on the efficacy and risks.

The ACCF/AHA/HRS guidelines do not specify the efficacy or the risks that justify a class I recommendation. Instead, they stipulate that the procedure be performed in experienced centers defined as performing more than $50 \mathrm{AF}$ catheter ablations per year although they acknowledge that this recommendation is not evidence based [1]. The 2010 Canadian

The opinions expressed are entirely those of Dr. Anderson and do not represent those of the JICE. From The Department of Cardiology, Marshfield Clinic, Marshfield, and the Department of Medicine, University of Wisconsin Medical School, Wisconsin, USA. Dr. Anderson has no financial, commercial, or industrial relationships related to this commentary.

K. P. Anderson $(\square)$

Department of Cardiology 2D2, Marshfield Clinic,

1000 North Oak Avenue,

Marshfield, WI 54449, USA

e-mail:wrenroad@hughes.net
Cardiovascular Society guidelines specify both an efficacy rate $(60-75 \%$ after one procedure and $75-90 \%$ after two procedures) and complication rates (overall $2-3 \%$, vascular access complication $1-2 \%$, cardiac perforation $0.5-1 \%$, thromboembolism $0.5-1 \%$ ) [2]. However, for evidence, they cite only a worldwide survey that reports a higher complication rate (see below) [3]. The 2010 Guidelines of the European Society of Cardiology suggest a more modest recommendation for catheter ablation of AF (level IIa) [4]. A table of clinical trials of catheter ablation is provided that indicates rates of $\mathrm{AF}$ free at 1 year from $56 \%$ to $87 \%$. A table describing specific complications is also provided including death $(0.7 \%)$, thromboembolism [0.93\% (transient ischemic attack-TIA $0.2 \%$, stroke $0.3 \%$ )], tamponade up to $6 \%$ of all procedures, and $0.8 \%$ with hypotension or cardiac arrest. There is, therefore, variance among the major American, Canadian, and European guidelines regarding the strength of recommendation for $\mathrm{AF}$ ablation, level or grade of evidence, and expectation for procedure experience, efficacy, and complications.

In a recent survey, major procedural complications of radiofrequency catheter ablation for AF occurred in $4.5 \%$ of 16,309 patients who underwent 20,825 procedures between 2003 and 2006 from 85 centers worldwide [3]. In this survey there were 25 deaths $(0.15 \%), 213$ cardiac tamponades $(1.31 \%), 25$ permanent diaphragmatic paralyses $(0.17 \%), 6$ atrial-esophageal fistulae $(0.04 \%), 37$ strokes $(0.23 \%), 115$ TIAs $(0.71 \%), 11$ valve damages $(0.07 \%)$, 152 femoral pseudoaneurysms $(0.93 \%), 88$ arterial-venous fistulae $(0.54 \%)$, and 48 pulmonary vein stenoses requiring intervention $(0.29 \%)$ and assorted other low-frequency but serious complications. Many clinicians would be reluctant to recommend a procedure to reduce symptoms with these many serious complications. The level of experience was not low as the median number of procedures per center was 245, well above the suggested minimum $50 \mathrm{AF}$ ablation procedures per year recommended by the ACCF/AHA/HRS 
guidelines [1]. The $4.5 \%$ rate in the most recent survey is even higher than the $4.0 \%$ rate found in the previous survey based on 12,830 procedures performed in 8,745 patients between 1995 and 2002, causing the investigators to conclude that complication rates do not appear to be decreased with experience [3]. On the other hand, the cited complication rate of $4.5 \%$ is a simple arithmetic mean which is not very informative and may not be a good estimate of the complication rate of the average center. Also, the investigators did not present a formal analysis of the role of experience in this survey. Moreover, the data in this survey were obtained from questionnaires sent to 521 centers, 85 of whom responded with sufficient completeness to be included in the survey. Therefore, the data could be biased by characteristics of ablation centers that had the interest and resources to complete the questionnaires.

Shah et al. performed a more "real world" survey of all 4,156 patients who underwent initial ablation for AF at nonfederal California hospitals between 2005 and 2008 [5]. Major complications during the index hospitalization occurred in $5.1 \%(n=211)$ of patients, and 9.4\% $(n=390)$ were rehospitalized within 30 days. Calculations assuming all events were captured, were independent and were not double counted indicate rates of death, stroke, and cardiac perforation or tamponade of $0.24 \%, 0.70 \%$, and $2.79 \%$, respectively. These rates are substantially higher than reported in the worldwide ablation survey [3]. Furthermore, death may be under reported as only in-hospital deaths were included.

Shah and colleagues performed an analysis that included a covariate for "recent procedure experience" defined as the number of cases completed during the year before the index $\mathrm{AF}$ ablation divided into quartiles. The mean number of procedural experiences ( \pm standard deviation) was $54.32 \pm$ 53.2. This variable demonstrated independent predictive value along with female sex, number of AF hospitalizations during prior year, and age for inpatient complications or allcause 30-day rehospitalizations. The experience variable was associated with a $57 \%$ increase in the odds of the outcome in the lowest compared with the highest quartile. Every five ablation increase in the number of procedures completed during the prior 12 months decreased the odds of the outcome by $2 \%$. This analysis suggests that the average number of AF ablation complications "in the real world" is too high, that 50 ablation procedures per year is not necessarily associated with a satisfactorily low level of complications, but that greater experience is associated with lower levels of complications. The large number of events and inclusive nature of this analysis adds value to the results. However, it must be recognized that estimates of clinical events based on administrative databases without appropriate audits have a substantial potential for errors [6].

Hoyt and colleagues examined the role of experience and other factors contributing to complications related to $\mathrm{AF}$ ablation at a renowned medical center in the USA [7]. The rate of major complications among 1,190 procedures performed in 931 patients between February 2001 and December 2010 was $4.7 \%$. There were 13 cardiac tamponades requiring pericardiocentesis $(1.1 \%), 9$ strokes $(0.8 \%)$, and no deaths. The authors note that only six $(0.5 \%)$ patients had permanent sequelae. The annual major complication rate decreased from $11.1 \%$ to $1.6 \%$ over the period of study. The investigators observed that the greatest decrease in the overall complication rate occurred between 2002 and 2003 (from $11.1 \%$ to nearly $6 \%$ judging from Fig. 1 in the article), during which time their institution performed more than 100 procedures which has been suggested as a threshold for identifying high-volume centers with lower complication rates [7]. However, it is evident from the data presented that the complication rate continued to decline with another halving between 2007 and 2008 (from nearly 6\% to about $3 \%$ after over 700 procedures) and with another near halving during the next 2 years to $1.6 \%$ in 2010 after over 1,000 cases. Hoyt and colleagues found that CHADS2 score $\geq 2$ and female sex were independent predictors of major complications, but an "experience" covariate was not included. The investigators examined the effect of experience by comparing outcomes of those operators who performed $\leq 30$ cases and those who performed $>30$ cases. No significant differences in major complication rates were identified. The investigators found that complication rates for operators who began ablations 2001-2005 trended higher (5.3\%) than for those who began ablations 2006-2010 (2.0\%), but the differences were not significant. Although specific statistical evidence was not provided, the investigators demonstrated a dramatic decline in complication rates during a nearly 10 -year period that could not be explained entirely by changes in procedures or equipment and did not appear to be diminished despite the participation of new operators with less experience. For this reason, the authors concluded that the decline of complications "correlates principally with institutional experience" [7].

In this issue of the journal, Winkle and colleagues report the experience of a private practice group operating at a community hospital that compliments the study of Hoyt and colleagues reporting from an academic medical center in an urban environment $[7,8]$. The numbers of patients $(1,125)$ and number of ablations $(1,504)$ and duration of inclusion $(2003$ 2010) are similar. During their first 2 years (2003 and 2004), Winkle and coworkers performed 43 ablations and report a major complication rate of $4.65 \%$, similar to the values reported in the worldwide survey and the overall experience of Hoyt et al. despite differences in methods of ascertainment $[3,7]$. During the next year, 2005, the investigators performed 79 ablations and the total (major plus minor) complication rate dropped from $9.3 \%$ to $5.1 \%$. This supports the observation of Hoyt et al. that 50-100 ablations are associated with a prominent decline in complications. However, also similar to Hoyt et al., major complications continued to decline to $1.05 \%$ by 
2010. Total complications decreased from $9.30 \%$ to $1.58 \%$ over time $(P=0.001)$. The investigators opined that the shape of the curve indicates a "long" learning curve of about 6 years (approximately 800 procedures based on computations from Table 2 of the article) to minimize ablation complications.

A striking finding from the study of Winkle et al. is that mean procedure times decreased by more than $50 \%$, from $256+49$ to $122+28 \mathrm{~min}$, and mean fluoroscopy times decreased from $134+29$ to $56+19 \mathrm{~min}$. The trajectory shows the greatest decline early in the experience but with continued decline each year. This is good news to patients, operators, and health care systems as it likely means lower duration under anesthesia, less radiation exposure to patients and operators, and greater efficiency.

The reasons for the observed changes cannot be determined with certainty. There was a gradual increase in the mean age of patients from 59 to 64 and an increase in the average CHADS2 score from $0.77+0.95$ to $1.15+1.08(P<0.0001)$. Based on the analyses of Shah et al. and Hoyt et al., as described above, these changes would be expected to increase, not decrease, the risk of major complications [5, 7]. Over time, more patients had comorbidities including hypertension, coronary artery disease, and prior strokes or TIAs. It seems unlikely that these factors would result in lower complication rates. There was no change in gender, number of antiarrhythmic drugs failed, duration of AF prior to ablation, body mass index, or left atrial size over time. There was a slight decrease in the number of patients with paroxysmal AF, which was borderline significant, a significant increase in the number with persistent $\mathrm{AF}$, and a significant decline in the number with long-standing persistent AF.

In January, 2006, the investigators changed to an open irrigated tip catheter. Because there were progressive reductions in complications and procedure times before and after this development, there is no strong evidence this had a major impact. During the study, the target activated clotting time was reduced from $>300$ to $<250 \mathrm{~s}$. This may have contributed to a decline in some complications such as the significant reduction in cardiac tamponade. It did not appear to result in more strokes or TIAs. However, it is of interest to note that the target ACT of Hoyt and colleagues was increased from 300 to 350 $400 \mathrm{~s}$ during the same time period as complications declined [7]. The large increase in ablations and the concomitant progressive decline in complications and procedure times and lack of alternative explanations lead to the conclusion that the most likely factor resulting in the decline in complications was the cumulative experience of the ablation team.

Another striking observation of Winkle and colleagues is that despite the improvements in equipment and the cumulative procedural experience, there were minimal gains in efficacy, as assessed by cumulative probability of freedom from AF after ablation. The investigators found a statistically significant year-to-year improvement in outcomes after the initial ablation in patients with paroxysmal AF. Most of the improvement appeared to occur up to 2006, whereas little improvement was evident after that year. There was also evidence of a trend for improvement for initial procedures in patients with persistent AF. However, most of the improvement occurred before 2007, with little improvement thereafter. For long-standing persistent AF, no improvement was discernible for first ablations. When success rates were examined after combined ablation procedures, no statistically significant patterns of improvement were identified with any of the three forms of AF examined. It is interesting to note that in the worldwide survey mentioned above, an increase in freedom from $\mathrm{AF}$ without need for antiarrhythmic drugs compared to the previous survey was observed, from $52 \%$ to $70 \%$, whereas overall proportions of freedom from $\mathrm{AF}$ were not improved (79.8\% to $80.0 \%$ ) [3].

Patients with AF may have such debilitating symptoms that they are willing to undergo ablation for AF despite significant risk even though the procedure will not prolong life or necessarily prevent future morbidity. Nevertheless, they are likely to have an expectation that the procedure will be successful and the chance for relief of symptoms outweighs the risks. Clinicians need to understand the probability of success and complications in order to inform patients and to make judgments for individual patients. Efficacy rates of $60-75 \%$ after one procedure and $75-90 \%$ after two procedures, as stated in the guidelines of the Canadian Cardiovascular Society are compatible with results from worldwide survey and the study of Winkle and colleagues $[3,8]$. In contrast, there is substantial variance for major complication rates and the average rates reported in the surveys reviewed above seem unacceptably high $(>4 \%)$ [3-5]. The reports of Hoyt and colleagues and Winkle and colleagues suggest that rates of major complications of less than $2 \%$ are achievable but may require the experience of hundreds of procedures. Perhaps a highvolume center should be defined as one with over 1,000 procedures. Fortunately, the data from Hoyt et al. suggest that the incorporation of less experienced operators does not significantly interfere with the decline in complication rate. This implies that it is the cumulative experience of the AF ablation center that is essential for reducing complications.

Recent surveys and reports of practice experience suggest that centers performing AF ablation should review their complications. Those with major complication rates greater than $4 \%$ should consider whether or not it is ethical for them to continue to offer the service. If the rate is between $4 \%$ and $2 \%$, AF ablation centers should execute a strategy to reduce the complication rate to less than $2 \%$. Accomplished ablation centers should consider public reporting of complication rates including enumeration of those that often result in permanent effects and those which are reversible but life threatening.

Winkle and colleagues have shown us a path for reducing the complication rate in AF ablation to a level that is well 
below averages published in large surveys and in a range that patients and clinicians are likely to find acceptable considering the benefits of the procedure. In addition, they provide evidence that should temper our expectations for improving procedural success based on current methods. Their opinion that new technologies such as robotic and magnetic navigation systems and cryoballoon ablation systems do not improve ablation outcomes may stimulate debate. However, practices and hospitals considering incorporation of new technology should carefully assess the learning curve to avoid slipping back on the experience trajectory that could result in increased complications or lower efficacy. Few would disagree with the conclusion of Winkle and colleagues that better understanding of the mechanisms of AF is the key to better clinical results.

\section{References}

1. Wann, L. S., Curtis, A. B., January, C. T., Ellenbogen, K. A., Lowe, J. E., Estes, N. A. M., 3rd, et al. (2011). 2011 ACCF/AHA/HRS focused update on the management of patients with atrial fibrillation (updating the 2006 guideline): A report of the American College of Cardiology Foundation/American Heart Association Task Force on Practice Guidelines. Journal of the American College of Cardiology, 57(11), e101-98.
2. Verma, A., Macle, L., Cox, J., Skanes, A. C., \& CCS Atrial Fibrillation Guidelines Committeee Canadian Cardiovascular Society. (2011). Atrial Fibrillation Guidelines 2010: Ablation for atrial fibrillation/atrial flutter. The Canadian Journal of Cardiology, 27, 60-66.

3. Cappato, R., Calkins, H., Chen, S. A., et al. (2010). Updated worldwide survey on the methods, efficacy, and safety of catheter ablation for human atrial fibrillation. Circulation. Arrhythmia and Electrophysiology, 3, 32-38.

4. Camm, A. J., Kirchhof, P., Lip, G. Y., Schotten, U., Savelieva, I., Ernst, S., et al. (2010). Guidelines for the management of atrial fibrillation: The Task Force for the Management of Atrial Fibrillation of the European Society of Cardiology (ESC). Europace, 12 (10), 1360-420.

5. Shah, R. U., Freeman, J. V., Shilane, D., Wang, P. J., Go, A. S., \& Hlatky, M. A. (2012). Procedural complications, rehospitalizations, and repeat procedures after catheter ablation for atrial fibrillation. Journal of the American College of Cardiology, 59, 143-9.

6. Anderson, K. P. (2009). Estimates of implantable cardioverterdefibrillator complications: Caveat emptor. Circulation, 119, 1069-71.

7. Hoyt, H., Bhonsale, A., Chilukuri, K., Alhumaid, F., Needleman, M., Edwards, D., et al. (2011). Complications arising from catheter ablation of atrial fibrillation: Temporal trends and predictors. Heart Rhythm, 12, 1869-74.

8. Winkle, R. A., Mead, R. H., Engel, G., Kong, M. H., \& Patrawala, R. A. (2012). Trends in atrial fibrillation ablation: Have we maximized the current paradigms? Journal of Interventional Cardiac Electrophysiology (in press). 